The Israeli Journal of Aquaculture IJA.73.2021.1446207, 15 pages

CCBY-NC-ND-4.0 • https://doi .org/10.46989/001c.27662

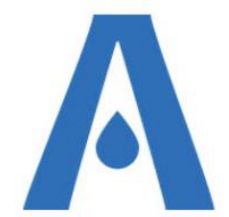

The $I J A$ is a peer-reviewed open-access, electronic journal, freely available without charge to users

Produced by the AquacultureHub non-profit Foundation Sale of $I J A$ papers is strictly forbidden

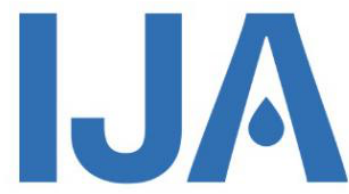

\title{
Transcriptome analysis of Marsupenaeus japonicus hepatopancreas during WSSV persistent infection
}

\author{
Minze Liao ${ }^{1+}$, Jichen Zhao ${ }^{1+}$, Zihao He ${ }^{1}$, Xieyan Chen ${ }^{1}$, Yuan Xue ${ }^{1}$, \\ Jianing Zhou ${ }^{1}$, Xinxin Long ${ }^{1}$, Chengbo Sun ${ }^{1}{ }^{2}$ 3* $^{*}$ \\ ${ }^{1}$ College of Fisheries, Guangdong Ocean University, Zhanjiang, Guangdong, P.R. China \\ 2 Guangdong Provincial Laboratory of Southern Marine Science and Engineering, \\ Zhanjiang, Guangdong, P.R. China \\ ${ }^{3}$ Guangdong Provincial Key Laboratory of Pathogenic Biology and Epidemiology for \\ Aquatic Economic Animals, Zhanjiang, Guangdong, P.R. China
}

Key words: white spot syndrome virus, Marsupenaeus japonicus, persistent infection, transcriptome analysis, immunity strategy

\begin{abstract}
White Spot Syndrome Virus (WSSV) can cause a large-scale death of cultured shrimp and significant damage to the shrimp farming industry. Marsupenaeus japonicus is one of the world's most important economically farmed shrimp. This study found that some M. japonicus survived the spontaneous outbreak of WSSV. Surprisingly, these virus-carrying shrimp showed no apparent illnesses or outbreaks of white spot disease in the subsequent cultivation, and their body size was substantially smaller than healthy shrimp, indicating a long-term fight between the host and the virus. To investigate this interesting phenomenon, we analyzed the transcriptomes of healthy shrimp and survived shrimp through the RNA-Seq platform, attempting to reveal the underlying molecular mechanism of the struggle between M. japonicus and WSSV. Transcriptional analysis showed that a total of 37,815 unigenes were assembled, with an average length of $1,193.34 \mathrm{bp}$ and N50 of 2,049 bp. In the KEGG pathway, enrichment analysis of DEGs pathways related to immunity, biosynthesis, and growth metabolism was enriched, including pentose phosphate pathway, glycerophospholipid metabolism, fatty acid biosynthesis, Wnt signaling pathway, biosynthesis of amino acids, ascorbate, and aldarate metabolism. Our data showed a delicate balance between M. japonicus and WSSV infection: On the one hand, WSSV infection can cause host metabolism and biosynthesis disorders in the host, and the virus consumes a portion of the material and energy required for shrimp average growth and reproduction. If WSSV infection persisted for a long time, then the growth rate of $M$. japonicus decreased. On the other hand, the host can regulate immune defense to resist subsequent viral infection. This study reveals the underlying molecular mechanism of a long-term battle of $M$. japonicus against WSSV infection, providing novel insights for preventing WSSV persistent infection in $M$. japonicus and other farmed shrimp species.
\end{abstract}

\footnotetext{
* Corresponding author: Chengbo Sun, suncb@gdou.edu.cn
}

+ These authors contributed equally to this work 


\section{Introduction}

Marsupenaeus japonicus is one of the most important cultivated economic shrimps along the Chinese coast. It is a kind of marine product with great commercial value and is widely popular with consumers because of its delicious meat and high nutritional value. Due to its exceptional capacity to adapt to changing environments, $M$. japonicus has become essential cultivation aquaculture in an increasing number of national and regional areas. Currently, large-scale production of $M$. japonicus has been established, and it is quickly expanding (Yuquan et al., 2014). In 2019, the mariculture area of this shrimp was 21,443 ha, and 50,968 tons of $M$. japonicus were harvested, resulting in substantial profits to shrimp farmers. However, viral infections limit the growth of $M$. japonicus aquaculture, posing significant challenges to shrimp farming ( $\mathrm{Li}$ et al., 2013). Among the reported shrimp viruses, the White spot syndrome virus (WSSV) is one of the most harmful viruses in the shrimp farming industry (Escobedo-Bonilla et al., 2010) and outbreaks frequently in many areas, resulting in massive economic losses. The interaction between shrimp and viruses is currently receiving much interest in WSSV research (Leu et al., 2013).

Shrimp and other arthropods are known to carry single to numerous viral viruses at low levels without showing symptoms of illness, allowing them to survive for extended periods with chronic infections (Flegel 2007, 2009). Flegel proposed the "viral accommodation" model in 2007 to investigate the interaction between the host and the virus: the shrimp generate an active adaptation mechanism to the viral pathogen, which results in reduced severity of viral infection by blocking viral triggered apoptosis, and enables shrimp to survive for a long time (Flegel 2007). Subsequently, Flegel proposed the immunological hypothesis based on the original theory: The shrimp may integrate the viral sequence into its genome to create immune-specific RNA (imRNA), which induces host RNA interference (RNAi), thereby blocking viral transmission and playing a crucial role in future persistent infection without clinical symptoms (Flegel 2009). However, the underlying molecular mechanisms of persistent infection in shrimp are still elusive.

With the emergence of various omics, transcriptomics has become an important element in understanding biological information. In recent years, many studies have been conducted to reveal the underlying molecular mechanisms that cause WSSV to be detrimental to crustaceans through transcriptomics (Rao et al., 2015), including Litopenaeus vannamei infected with WSSV (Santos et al., 2020) and Macrobrachium Rosenbergii infected with WSSV (Rao et al., 2015). These studies have identified genetic factors and immune signaling pathways that may be implicated in resistance to WSSV and provided additional insights into the prevention and treatment of WSSV. However, the current studies on WSSV of shrimp have mainly focused on acute and latent infections, with no comprehensive reports of WSSV persistent infection so far.

In the present study, we employed the RNA-Seq platform to compare the transcriptional level of $M$. japonicus hepatopancreas between the WSSV virus-carrying survival and the virus-free healthy groups. The hepatopancreas was chosen as it can produce immune proteins such as hemocyanin and lectin, which play a crucial role in the immune defense of shrimp (Song et al., 2010). The study aimed to shed light on the molecular mechanism that M. japonicus fight off WSSV persistent infection after its spontaneous outbreaks and provide the scientific basis for better understanding the interaction between M. japonicus and WSSV.

\section{Detection of WSSV using PCR}

\section{Materials and Methods}

Before the sample collection, the shrimp from each healthy group and survival group were randomly selected for PCR amplification to confirm WSSV was not present in the healthy group. According to the manufacturer's protocol, total DNA from hepatopancreas was extracted with Marine Animal Genomic DNA Kit (Transgen, Beijing, China). PCR primers for WSSV detection were designed with software Primer 5.0 (Table 1). The $20 \mu \mathrm{L}$ polymerase chain reaction system contained PrimeSTAR Max Premix $(2 \times)$ (TaKaRa, Beijing, China) $10 \mu \mathrm{L}$, each primer $1 \mu \mathrm{L}$ (ie1-F and ie1-R), total DNA $1 \mu \mathrm{L}$, and ddH2O 7 $\mu \mathrm{L}$. The PCR was performed at $95^{\circ} \mathrm{C}$ for $3 \mathrm{~min}, 35$ cycles of $95^{\circ} \mathrm{C}$ for $30 \mathrm{~s}, 60^{\circ} \mathrm{C}$ for $50 \mathrm{~s}$ 
and $72{ }^{\circ} \mathrm{C}$ for $30 \mathrm{~s}$, ending at $72{ }^{\circ} \mathrm{C}$ for $1 \mathrm{~min}$. Furthermore, $10 \mu \mathrm{L}$ amplification product for PCR was pipetted and analyzed in a $1.5 \%$ agarose gel.

Table 1 Primers used for PCR and qPCR.

\begin{tabular}{|c|c|c|}
\hline No. & Primer names & Sequences $\left(5^{\prime}-3^{\prime}\right)$ \\
\hline \multicolumn{3}{|c|}{ PCR } \\
\hline 1 & ie1-F & ATGGCCTTTAATTTTTCAAGA \\
\hline 2 & ie1-R & ACAAAGAATCCAGAAATCTC \\
\hline \multicolumn{3}{|c|}{ qPCR } \\
\hline 3 & c-type lysozyme-F & CGTGTCCGAAGGTCTTTGAG \\
\hline 4 & c-type lysozyme-R & CCTGGTTCCGTGTTATTGGT \\
\hline 5 & trehalose transporter- $\mathrm{F}$ & GTCAGGCTCGTCAGCACTC \\
\hline 6 & trehalose transporter- $\mathrm{R}$ & GGGTTTGTGGTAGCCGTTT \\
\hline 7 & carbohydrate sulfotransferase $3-\mathrm{F}$ & ACACCCTGACCAAGTATCGC \\
\hline 8 & carbohydrate sulfotransferase 3-R & TACATCCCGTTGTCCCTCC \\
\hline 9 & WD repeat-containing protein $61-\mathrm{F}$ & ACCAGAGCAACGGACAACC \\
\hline 10 & WD repeat-containing protein $61-R$ & CATGGAACCAGAGGCAAGT \\
\hline 11 & C-type lectin 2-F & CTCTGTGCTCAGCCATCGG \\
\hline 12 & C-type lectin 2-R & CCACGCCTCGGTATCCTTC \\
\hline 13 & FEM-1-F & ACTTGGAGCAGGTGGGATC \\
\hline 14 & FEM-1-R & AGTTGGTCTTCGTTGTGGC \\
\hline 15 & protein serine threonine kinase- $F$ & GGCAGAAAGGTCATCAAGC \\
\hline 16 & protein serine threonine kinase- $\mathrm{R}$ & TCGGGTTCCATCAAAGTCA \\
\hline 17 & potassium channel subfamily $\mathrm{K}$ member- $\mathrm{F}$ & GTGGAGCGGCATAGCAGT \\
\hline 18 & potassium channel subfamily $\mathrm{K}$ member- $\mathrm{R}$ & ССТСАAССАССТGTAAATCG \\
\hline 19 & dLp/HDL-BGBP precursor-F & ACTCCGTGACGACTTCTACCG \\
\hline 20 & dLp/HDL-BGBP precursor-R & CATCAATGTGGGCGTGGG \\
\hline 21 & casein kinase I-F & CGTTCCCTTGGGCTTCTGT \\
\hline 22 & casein kinase I-R & GCCGAGGTCGAGGATGTAC \\
\hline 23 & EF1a-F & GGAACTGGAGGCAGGACC \\
\hline 24 & EF1a-R & AGCCACCGTTTTGCTTCAT \\
\hline
\end{tabular}

\section{Shrimp rearing conditions and sample collection}

In this study, healthy and vigorous larval M. japonicus were divided into two groups. The farming conditions were consistent with the previous article description: salinity was $22.46 \pm 1.76 \mathrm{ppt}$, $\mathrm{pH}$ was $7.72 \pm 0.54$, and temperature was $28.3 \pm 3.5^{\circ} \mathrm{C}$, respectively. The stocking density was 50 individuals $/ \mathrm{m}^{2}$, and artificial diet was used to feed the shrimp three times a day (He et al., 2020). After 97 days of shrimp cultivation, we found that one M. japonicus group had a spontaneous outbreak of WSSV without artificial interference. The shrimp in this group had obvious symptoms of white spot syndrome, and the results of PCR detection showed that the shrimp was positive for WSSV and negative for IHHNV and DIV1. During the WSSV flare-up, we treated the pond suffering from disease by increasing the quantity of exchanged water daily and removing the dead shrimp. In 11 days after the outbreak of WSSV, there were no more dead shrimp, and the surviving shrimp had no visible symptoms of white spot syndrome disease in the WSSV spontaneous outbreak group. No disease occurred in the healthy group during the cultivation period, and the shrimp grew well. The cultivation conditions of the healthy group and the WSSV spontaneous outbreaks group were the same. Our previous studies have showed that at the end of feeding, the healthy shrimp had the final weight $(11.64 \pm 0.51 \mathrm{~g})$ and final length $(9.63 \pm 0.17 \mathrm{~cm})$ significantly higher than the WSSV-survived shrimp with the final weight $(10.01 \pm 0.56 \mathrm{~g})$ and final length $(9.13 \pm 0.25 \mathrm{~cm})(p<0.01)($ He et al., 2020$)$.

The sample collection was carried out under aseptic conditions. Nine shrimp were randomly selected from the WSSV-survived group and the healthy group, respectively. To reduce individual differences, the hepatopancreas of three shrimp was put into a $1.5 \mathrm{~mL}$ 
tube on liquid nitrogen as one sample, which was immediately placed into liquid nitrogen. The tissue was then stored at $-80^{\circ} \mathrm{C}$ until RNA was extracted.

Total RNA extraction, library preparation, and transcriptome sequencing

The total RNA from the healthy group and WSSV survival group samples were extracted with Up Plus RNA Kit (Transgen, Beijing, China) following the manufacturer's protocol. The concentration of RNA samples was checked using SimpliNano (GE Healthcare, USA). PolyToligo-attached magnetic beads were used to purify mRNA from pooled RNA. Randomly broke the mRNA into short fragments by the addition of a fragmentation buffer. Subsequently, the first-strand cDNA was synthesized with mRNA as a template, and MMuLV Reverse Transcriptase and random hexamer primers, DNA Polymerase I, and RNase $\mathrm{H}$ were added to synthesize second-strand cDNA. After adenylation at the $3^{\prime}$ end of DNA fragments, it connected NEBNext Adaptor with the hairpin loop structure. The cDNA fragments of preferentially 300-400 bp in length were selected with the AMPure XP system (Beckman Coulter, Beverly, USA).

This study, constructed of six CDNA libraries via PCR amplification and enrichment: three libraries (T01, T02, T03) from healthy groups and three libraries (T04, T05, T06) from WSSV-carrying survival groups. Finally, the library preparations were sequenced on an Illumina Hiseq 2000 platform by Biomarker Technologies (Beijing, China).

\section{Transcriptome assembly and functional gene annotation}

Before the assembly, raw reads were filtered to remove the connector sequences, lowquality reads ( $\mathrm{Q} 30>85 \%$ ), and ambiguous reads (' $\mathrm{N}$ ' content $>10 \%$ ) using trimmomatic software (v0.36). After obtaining the high-quality sequencing data, it was assembled with the software Trinity software v2.5.1. TGICLI software v2.1 was used to remove redundant sequences and generate unigenes based on the sequence similarity and length. BLAST software (E-value $\leq 1 e-5$ ) was used to align unigene sequences to five available databases, including NR (ftp://ftp.ncbi.nih.gov/blast/db/), Swiss-Prot (http://www.uniprot.org/), GO (http://www.geneontology.org/), KOG (http://www.ncbi.nlm.nih.gov/KOG/) and KEGG (http://www.genome.jp/kegg/).

\section{Differentially expressed genes (DEGs) and analysis of enrichment}

RSEM (RNA-Seq by Expectation Maximization) software package was used to estimate the gene expression level for each sample. The FPKM (Fragments Per Kb per Million reads) value was used to indicate expression abundance. In this study, FDR (False Discovery Rate) was used as a key indicator for the screening of DEGs (differentially expressed genes), and genes with FDR $<0.05$ and $\mid \log 2$ (fold change) $\mid \geq 1$ were considered to be DEGs. The DEGs sets between the above healthy group and the WSSV survival group were obtained by the DESeq2 method (Love et al., 2014). Moreover, KEGG and GO were also used for the DEGs pathway and GO enrichment analysis, respectively.

\section{Verification by quantitative $R T$ - $q P C R$}

Ten randomly selected DEGs under the WSSV persistent infection are analyzed by RTqPCR, including five up-regulated and five down-regulated genes. And then, gene-specific primers were designed on the software Primer 5.0. Primer information is listed in Table 1. Before the Real-time PCR experiment, the isolated RNA was reverse-transcripted into highquality cDNA using a 5X All-In-One RT Master Mix (Applied Biological Materials, Vancouver, Canada). RT-qPCR was carried out using the SYBR ${ }^{\circledR}$ Premix EX Taq ${ }^{\mathrm{TM}}$ II (Tli RNase H Plus - Takara Bio, Japan) kit. The reaction system was carried out in a $20 \mu \mathrm{L}$ total volume, which follows as $10 \mu \mathrm{L}$ of $2 \times$ SYBR Green PCR buffer, $1 \mu \mathrm{L}$ of upstream primer, $1 \mu \mathrm{L}$ of downstream primer, $1 \mu \mathrm{L}$ of diluted cDNA, and $7 \mu \mathrm{L}$ of ddH2O. The amplification program was set to $95^{\circ} \mathrm{C}$ for $2 \mathrm{~min}, 40$ cycles of $95^{\circ} \mathrm{C}$ for $15 \mathrm{~s}$, and holding at $60{ }^{\circ} \mathrm{C}$ for $30 \mathrm{~s}$. All selected DEGs were verified by RT-qPCR using the CFX Connect ${ }^{\mathrm{TM}}$ Real-Time system (BioRad, USA). Each sample was set up for three biological replicates, and each reaction was set up for three technical replicates. The EF1a of M. japonicus was served as an internal 
control and normalized the expression level of each gene. The relative gene expression level was calculated using the $2^{-\triangle \Delta C T}$ method.

PCR detection of WSSV

\section{Results}

Just as Figure 1 shown, the PCR amplification results showed that a 675 bp DNA sequence corresponding to WSSV was generated in M. japonicus hepatopancreas samples. Compared to the shrimp that WSSV infected acute stage, survived shrimp carried low viral loads in their body. On the contrary, there were no visible viral loads of the WSSV in the healthy shrimp.

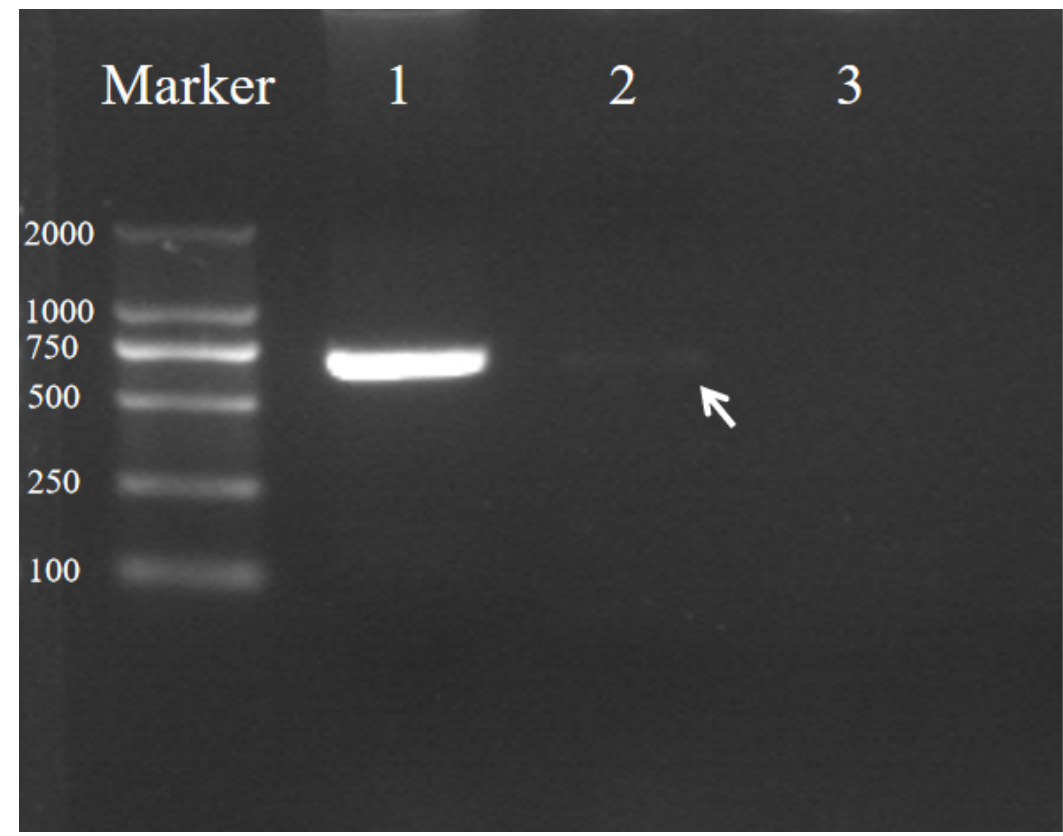

Figure 1 WSSV detection of $M$. japonicus hepatopancreas using PCR method. Marker: DL2000 molecular mass marker; Lane 1: PCR amplified products with DNA template samples extracted from WSSV acute infection shrimp; Lane 2: PCR amplified products with DNA template samples extracted from WSSV-survived shrimp; Lane 3: PCR amplified products with DNA template samples extracted from healthy shrimp.

Transcriptome sequencing and assembly

The Illumina HiSeq 2000 sequencing platform was applied for transcriptome sequencing on M. japonicus hepatopancreas samples from a healthy group (T01, T02, T03) and WSSV survival group (T04, T05, T06). After eliminating low-quality sequences, the healthy groups and WSSV-infected groups yielded $85,773,942$ and 106,995,442 clean reads, respectively. The healthy group got $25,628,750,012$ nucleotides, whereas the WSSV survival group obtained $31,966,256,556$ nucleotides. The quality analysis showed that the Q30 of each sample was located between $94.50 \%$ and $95.28 \%$ (Table 2 ).

All sequencing reads are submitted into the NCBI Sequence Read Archive (SRA, http://www.ncbi.nlm.nih.gov/Traces/sra), where they may be found under accession number PRJNA732090. Furthermore, using Trinity software to de novo assemble the clean reads, 79,188 transcripts with an average length of $1,667.52 \mathrm{bp}$ were produced. These transcripts were then spliced into 37,815 unigenes with an average 1,193.94 bp (N50 Length $=2,049 \mathrm{bp}$ ). Among these unigenes, the number of unigenes with length intervals ranging from 300 to 500 bp was the most, with 14,811. The unigenes with length interval longer than $2 \mathrm{kbp}$ was the least, with 6,439 (Table 3 and Figure 2). 
Table 2 Summary of reads and data for sequencing in six libraries.

\begin{tabular}{cccccc}
\hline Sample & & Read Number Sum & Base Number Sum & GC Content (\%) & $\% \geq Q 30$ \\
\hline \multirow{3}{*}{ Healthy group } & T01 & $26,260,114$ & $7,848,918,130$ & $50.40 \%$ & $95.28 \%$ \\
& T02 & $25,173,987$ & $7,523,346,374$ & $49.54 \%$ & $95.17 \%$ \\
& T03 & $34,339,841$ & $10,256,485,508$ & $50.20 \%$ & $95.28 \%$ \\
\multirow{3}{*}{ WSSV-infected } & T04 & $34,010,477$ & $10,159,780,522$ & $50.75 \%$ & $95.10 \%$ \\
& T05 & $35,879,123$ & $10,726,207,938$ & $50.44 \%$ & $94.50 \%$ \\
& T06 & $37,105,842$ & $11,080,268,096$ & $51.07 \%$ & $95.28 \%$ \\
\hline
\end{tabular}

Table 3 Statistical results of de novo assembly of transcriptomic profiles of M. japonicus.

\begin{tabular}{ccc}
\hline Length Range & Transcript & Unigene \\
\hline $300-500$ & $20,221(25.54 \%)$ & $14,811(39.17 \%)$ \\
$500-1000$ & $18,446(23.29 \%)$ & $9,765(25.82 \%)$ \\
$1000-2000$ & $18,076(22.83 \%)$ & $6,799(17.98 \%)$ \\
$>2000$ & $22,444(28.34 \%)$ & $6,439(17.03 \%)$ \\
Total Number & 79,188 & 37,815 \\
Total Length & $132,047,536$ & $45,148,928$ \\
N50 Length & 2,804 & 2,049 \\
Mean Length & $1,667.52$ & $1,193.94$ \\
\hline
\end{tabular}

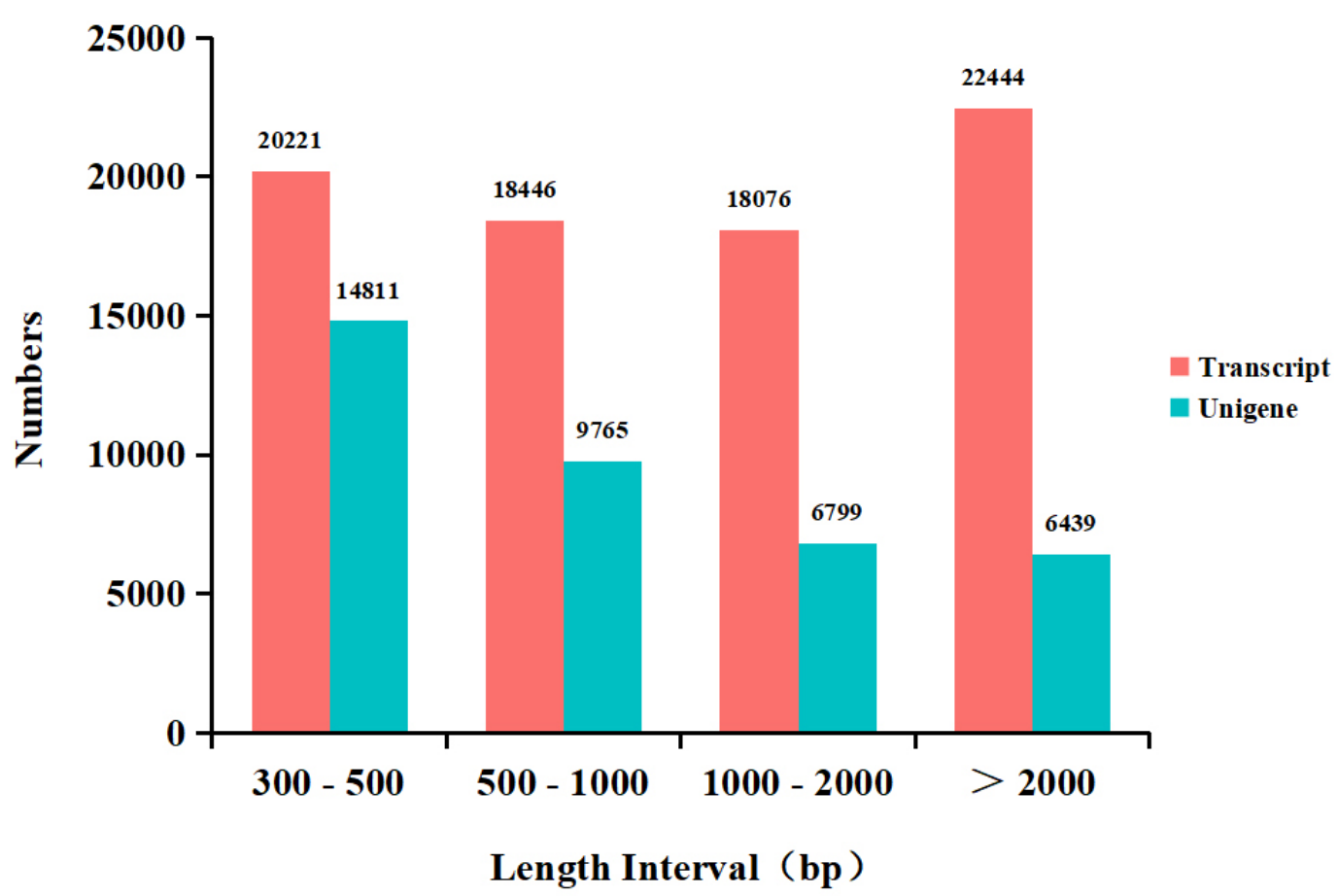

Figure 2 Statistical analysis of sequence length detected in $M$. japonicus hepatopancreas transcriptome. The $y$-axis shows the number of unigenes or transcripts; The $x$-axis shows the interval length.

Functional annotation and classification of M. japonicus transcriptome

BLAST software (Altschul et al., 1997) was used to compare unigene sequences with $\mathrm{Nr}$, Swiss-Prot, GO, KOG, and KEGG databases. The results showed that 11,909 assembled unigenes were annotated in the $\mathrm{Nr}$ database, 7,442 unigenes in the Swissprot database, 4,735 unigenes in the GO database, 8,529 unigenes in KOG database, and 6,492 unigenes in KEGG database, respectively (Figure $\mathbf{3}$ ). 

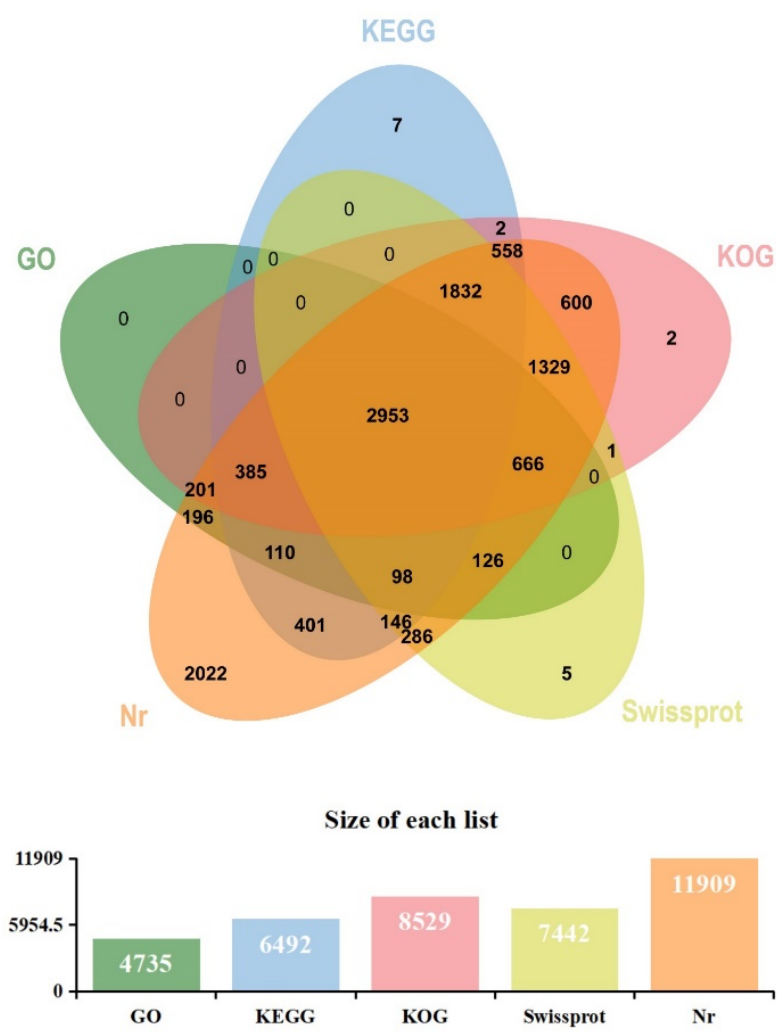

Figure 3 Statistics on the successful annotation of unigenes in each database.

In addition, $\mathrm{Nr}$ annotation showed that more than $60.82 \%$ of the unigenes matched with the sequences of ten various species: Hyalella azteca (35.75\%), Cryptotermes secundus $(6.16 \%)$, Zootermopsis nevadensis (4.46\%), Limulus polyphemus $(2.90 \%)$, Litopenaeus vannamei $(2.19 \%)$, Marsupenaeus japonicus $(2.07 \%)$, Centruroides sculpturatus (2.05\%), Daphnia pulex (1.91\%), Lingula anatina $(1.71 \%)$ and Penaeus monodon (1.62\%) (Figure 4).

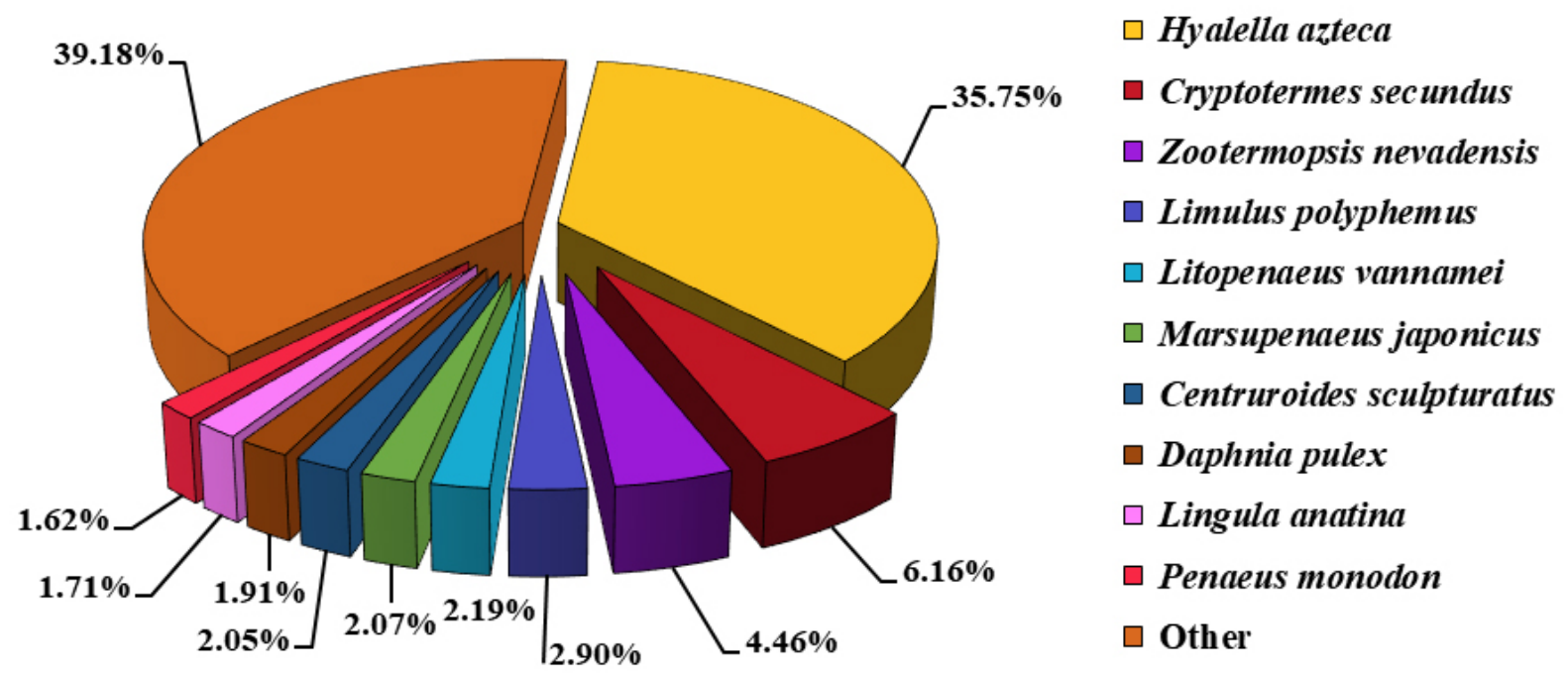

Figure 4 Species distribution of $M$. japonicus unigenes annotated in $\mathrm{Nr}$ database. 
The GO functional annotation showed that 4,735 unigenes aligned to the GO database were enriched in $48 \mathrm{GO}$ terms, divided into three categories: cellular component, molecular functions, and biological process. In the category of "biological processes", the largest number of unigenes were participating in "cellular process" and "metabolic process". Most unigenes in the "cellular component" category were involved in "cell part" and "cell". As for the "molecular functions" category, "catalytic activity" and "binding" were the dominant groups (Figure 5).

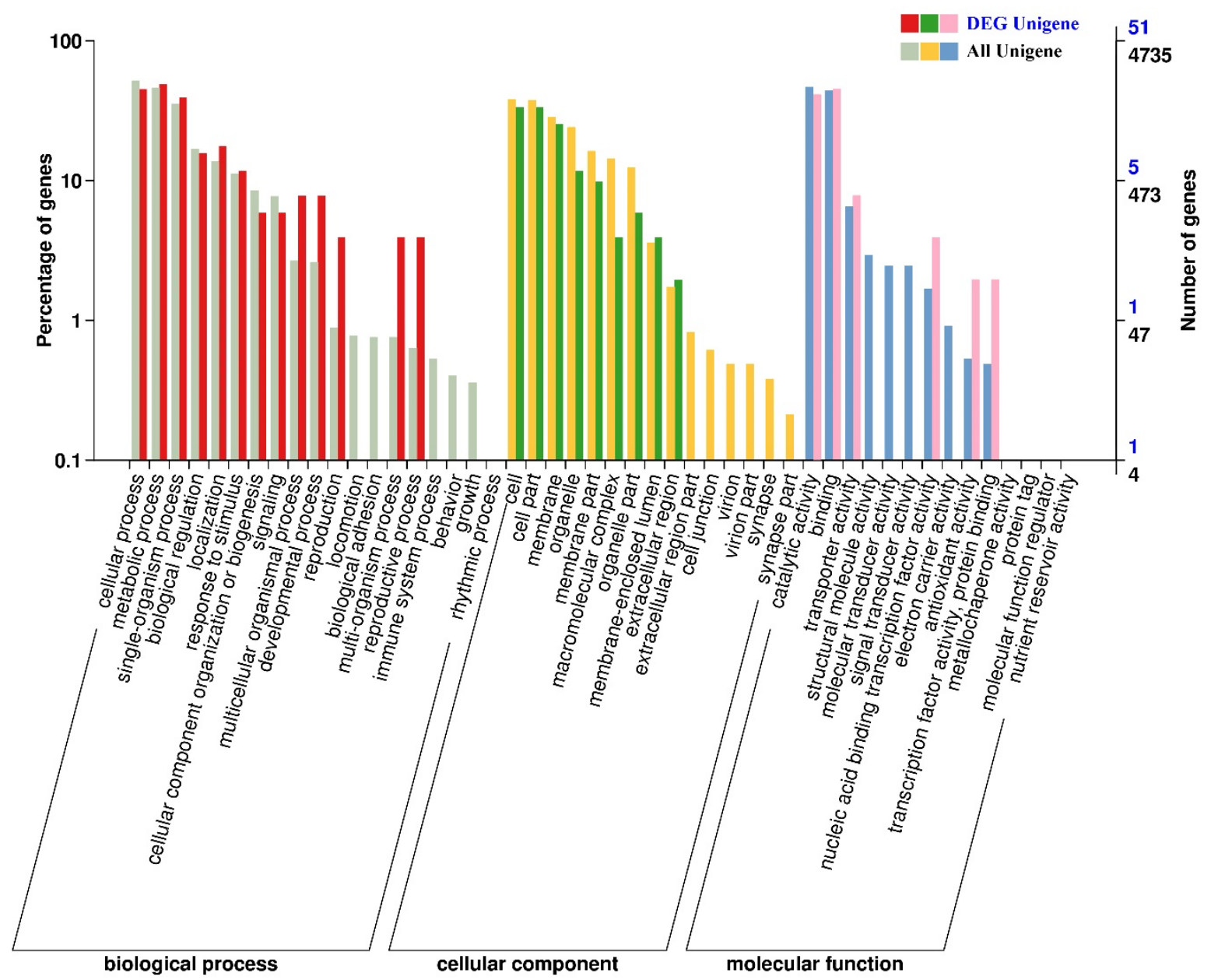

Figure 5 Gene ontology (GO) annotation of M. japonicus hepatopancreas. The x-axis represents GO functional classification. The $y$-axis on the left represents the percentage of annotated genes and the $y$-axis on the right represents number of annotated genes: the blue and black numbers represents the number of DEGs and the number of AllGenes, respectively.

KOG classification is important for functional annotation and gene studies. In this study, 8,529 unigenes were annotated to the KOG database, distributed in 25 categories. Among the functional classification categories, the "Signal transduction mechanisms" $(18.37 \%)$ represented the largest group, "General function prediction only" (17.35\%) and "Posttranslational modification, protein turnover, chaperones" $(8.16 \%)$ were next to it (Figure 6). 


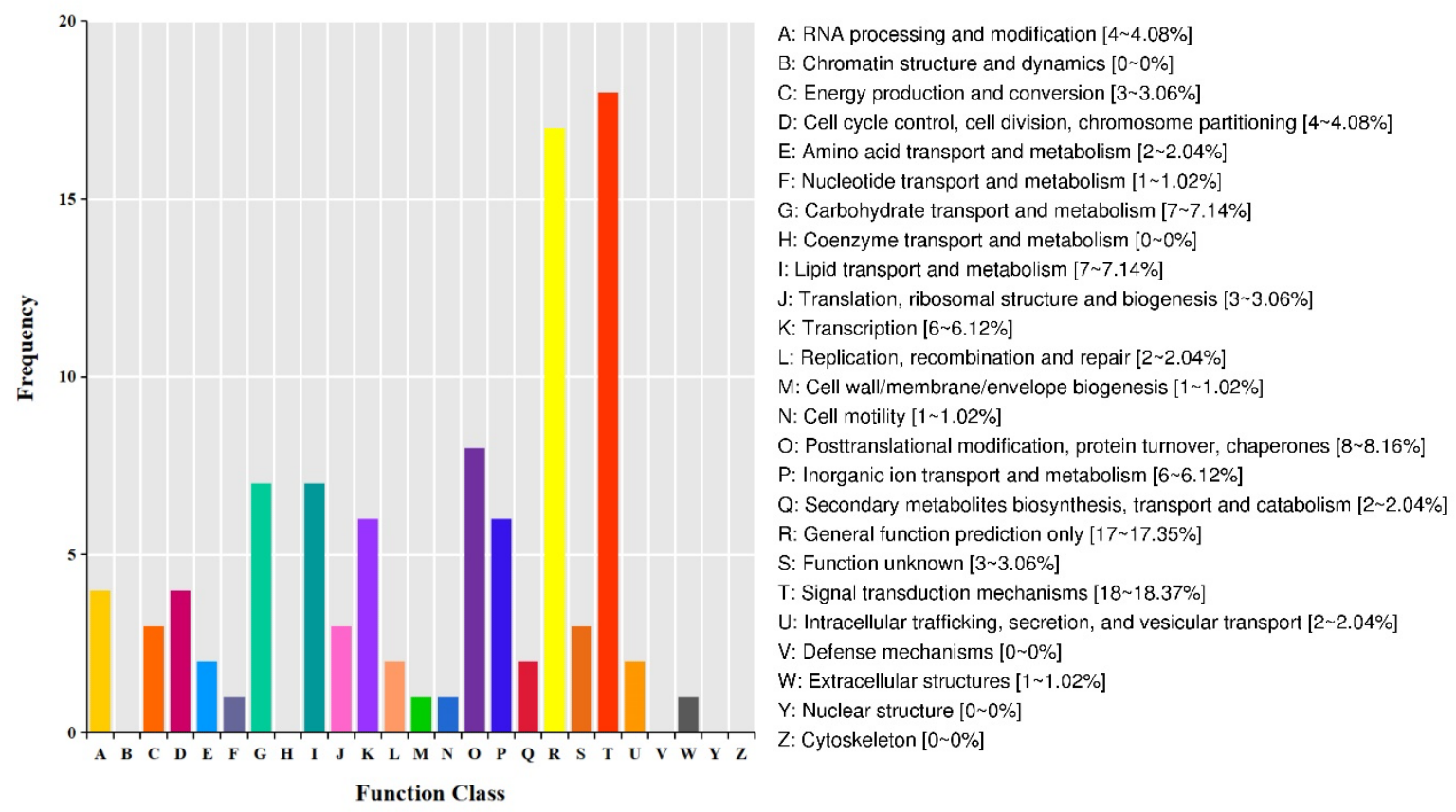

Figure 6 KOG functional annotation in the transcriptome of the hepatopancreas in M. japonicus. Xaxis stands for the functional categories; $\mathrm{Y}$-axis indicates the frequency.

\section{Functional characterization and identification of DEGs}

We selected DEGs in M. japonicus hepatopancreas transcripts of WSSV-survived and healthy group using FDR $<0.05$ and FC $\geq 2$ as filtering criteria. The results revealed that a total of 269 DEGs were screened, including 151 up-regulated genes and 118 downregulated genes (Figure $\mathbf{7}$ ).

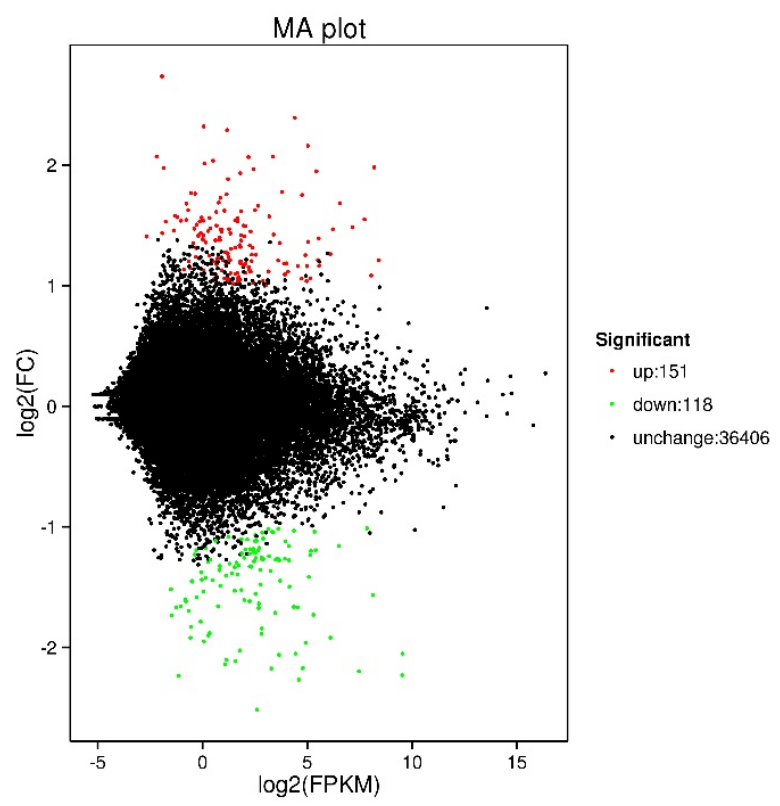

Figure 7 MA plot between differential expression genes in the group infected with WSSV vs. healthy group. The $x$-axis indicates the logarithmic value of the mean expression quantity in the two samples. The $y$-axis indicates the logarithm of the multiple gene expression difference between the two samples, which measures gene expression. The green and red dots represent genes with significant differences in expression, green represents down-regulated gene expression, red represents up-regulated gene expression, and black dots represent genes with no significant differences in expression. 
To further understand the function of DEGs, we annotated all the detected DEGs through GO and KEGG databases. In the GO functional analysis, a total of 28 DEGs were enriched into three main functional categories in the GO database, that is, biological process (13 subcategories), cellular component (9 subcategories), and molecular function (6 subcategories) (Figure 5). Among the category of "biological process", "metabolic process", "cellular process," and "single-organism process" were the three enrichment subclasses with the highest enrichment of DEGs. In the category of "cellular components", the corresponding DEGs were mainly enriched in "cells" and "cellular parts". The most subclasses of DEGs in "molecular functions" were "binding" and "catalytic activity". The statistical enrichment of DEGs in the KEGG pathway was examined through the analysis of differentially expressed genes. As shown in Figure 8, the top 20 pathways with the most reliable enrichment significance (the lowest $Q$ value) were selected to show the results, including the pentose phosphate pathway, glycerophospholipid metabolism, fatty acid biosynthesis, wnt signaling pathway, amino acid biosynthesis. Furthermore, some common immune genes related to immune were detected in the enrichment analysis of KEGG, such as heat shock protein 90 and C-type lectin 2 (Table 4).

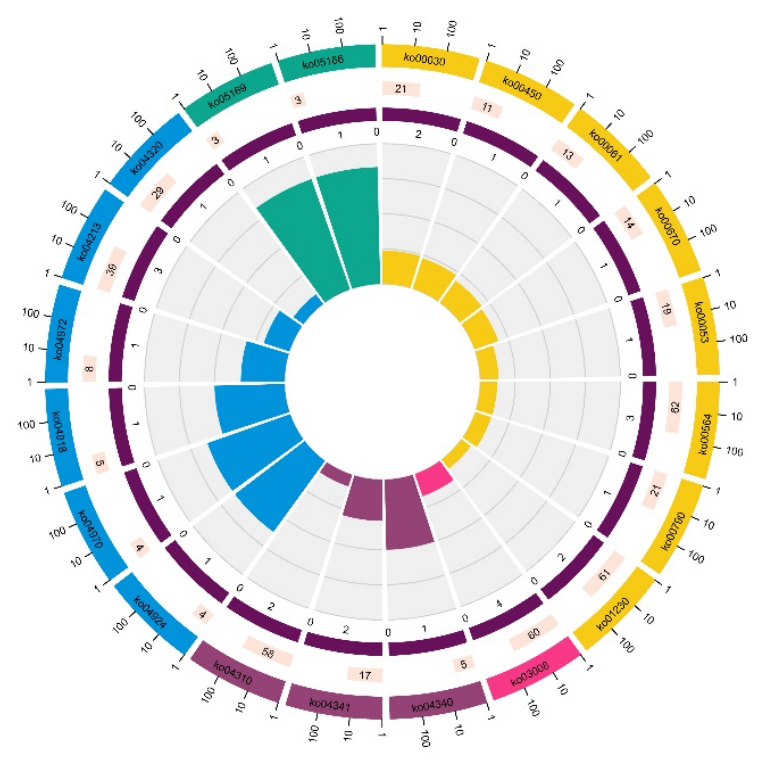

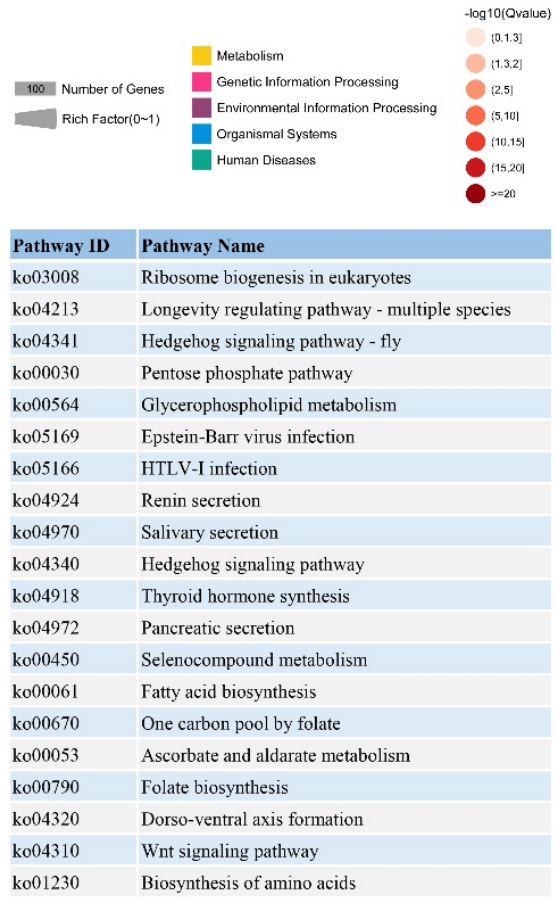

Figure 8 The results of DEGS in KEGG pathway enrichment analysis. Four laps from the outside to the inside, the first lap represents top 20 KEGG term, and different colors represent different classifications, with the number of the genes corresponding to the outer lap. The second lap represents the number of genes in the genome background and the $q$-value for enrichment of the DEGs in specific biological process. The more genes, the longer the bars. The third lap represents the total number of DEGs. The fourth lap represents the enrichment factor of each KEGG term. 
Table 4 DEGs connected with immune response in the process of persistent WSSV infection.

\begin{tabular}{|c|c|c|c|}
\hline Category or gene ID & Gene description & Species & $\log 2(F C)$ \\
\hline \multicolumn{4}{|c|}{ Protein processing in endoplasmic reticulum } \\
\hline c48367.graph_c2 & heat shock protein 21 & $\begin{array}{l}\text { Macrobrachium } \\
\text { rosenbergii }\end{array}$ & 1.53 \\
\hline c53576.graph_c0 & heat shock protein 90 & Marsupenaeus japonicus & 1.68 \\
\hline c53631.graph_c0 & heat shock protein 21 & $\begin{array}{l}\text { Macrobrachium } \\
\text { rosenbergii }\end{array}$ & 1.38 \\
\hline c54311.graph_c0 & heat shock protein 21 & $\begin{array}{l}\text { Macrobrachium } \\
\text { rosenbergii }\end{array}$ & 1.47 \\
\hline \multicolumn{4}{|c|}{ Wnt signaling pathway } \\
\hline c42977.graph_c0 & casein kinase I & Drosophila hydei & -1.66 \\
\hline c50973.graph_c0 & $\begin{array}{l}\text { guanine nucleotide-binding protein } \\
\text { subunit alpha homolog }\end{array}$ & Dinoponera quadriceps & 1.53 \\
\hline \multicolumn{4}{|c|}{ Pentose phosphate pathway } \\
\hline c35015.graph_c0 & fructose 1,6-biphosphate-aldolase $A$ & $\begin{array}{l}\text { Fenneropenaeus } \\
\text { chinensis }\end{array}$ & 1.17 \\
\hline \multicolumn{4}{|c|}{ MAPK signaling pathway - fly } \\
\hline \multicolumn{4}{|c|}{ Hippo signaling pathway - fly } \\
\hline \multicolumn{4}{|c|}{ FoxO signaling pathway } \\
\hline c50973.graph_c0 & G1/S-specific cyclin-D2-like & $\begin{array}{l}\text { Zootermopsis } \\
\text { nevadensis }\end{array}$ & 1.53 \\
\hline \multicolumn{4}{|l|}{ Lysosome } \\
\hline $\begin{array}{l}\text { c55477.graph_c0 } \\
\text { Thyroid hormone s }\end{array}$ & sialin-like & Hyalella azteca & -1.27 \\
\hline c45342.graph_c0 & C-type lectin 2 & Marsupenaeus japonicus & 1.12 \\
\hline
\end{tabular}

Validation of RNA-Sequencing transcriptome data via Real-time PCR

To further verify the authenticity of RNA sequencing results, ten genes were randomly selected for real-time PCR, including five up-regulated and five down-regulated genes. The results showed that the expression of the selected genes was like RNA-sequencing results in the real-time PCR analysis, indicating that the transcriptome data was reliable (Figure 9).

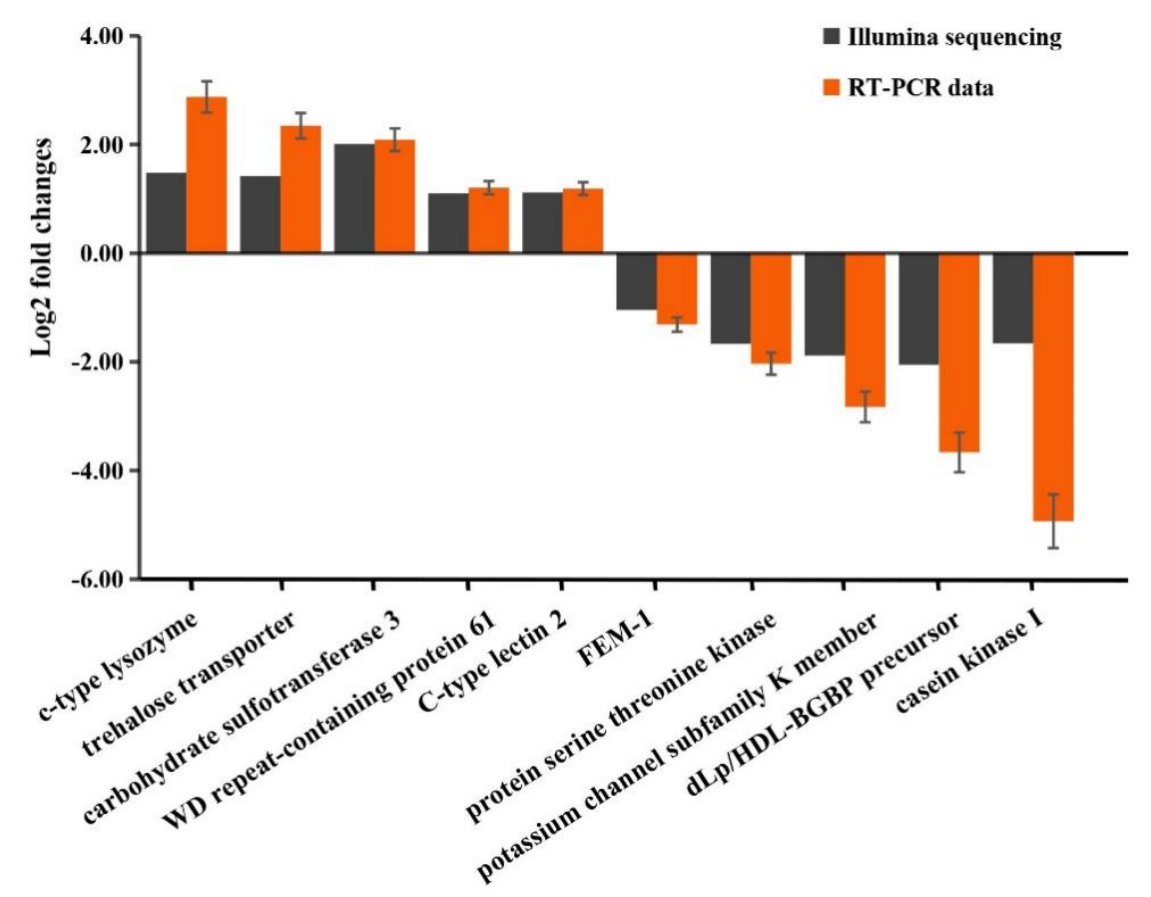

Figure 9 Comparison of RNA-Seq and RT-qPCR expression data of 10 randomly selected genes 


\section{Discussion}

Due to its characteristics of a short production cycle and significant economic value, marine shrimp cultivation occupies an essential position in aquaculture. However, with the fast expansion of intensive farming for shrimp, in recent years, the stocking density and pollution in the cultivation process have surpassed the environmental bearing capacity. As a result, the breeding environment was destroyed, resulting in frequent outbreaks of viral infections. The primary pathogen, WSSV, is responsible for outbreaks of epidemic diseases in shrimp (Lightner 2011). In recent years, the large-scale outbreak of WSSV has reduced shrimp production, leading to significant economic losses. Currently, the understanding of the mechanisms of host immune responses to persistent WSSV infection is still very limited. This study performed transcriptome analysis on WSSV persistently infected M. japonicus, revealing the molecular mechanism of the long-term battle between WSSV and $M$. japonicus. Notably, our previous studies have found that members of Cyanobacteria in the rearing water with WSSV outbreak group were quite abundant, some harmful cyanobacteria aggravate the spontaneous outbreak of WSSV, enhance the toxic effects and mortality (He et al., 2020), which could be the main primary cause of WSSV outbreaks.

KEGG pathway enrichment analysis of DEGs showed that certain pathways related to host immunity and viral infection were enriched. In the aspect of host immunity, the Wnt signaling pathway and ascorbate metabolism were activated. Studies have shown that the Wnt signaling pathway regulates many important biological processes in organisms, especially cell proliferation and apoptosis (Clevers et al., 2012). George et al. detected that Wnt ligands regulate the function of various immune cells to enhance the host's defense against human tuberculosis (George et al., 2008). A study found that Schneider line 2 cells (S2 cells) in the phagocytic cell line could engulf WSSV virions, while the phagocytic function of S2 cells was regulated by the Wnt signaling pathway (Zhu et al., 2013). These reports highlight the important role of the Wnt signaling pathway in host immunity against viruses. In shrimp, vitamin C (VC; ascorbic acid) improves survival and development rates, and it also helps to enhance the immune system (Tewary et al., 2008). Wang et al. discovered that adding VC to the Penaeus chinensis diet may enhance immunoglobulin and prophenoloxidase activity in shrimp serum (Weiqing et al., 2002). The latter was the shrimp key enzyme inactivating melanization (Tassanakajon et al., 2018). In DEGs analysis, our results revealed that heat shock protein 90 and C-type lectin 2 were upregulated in persistent WSSV infection. Some studies showed that Hsp90 (heat shock protein 90) is a protective protein. Hsp90 was synthesized in large amounts to help each cell maintain regular physiological activity when the host's living conditions suffer mutated (Sato et al., 2000). Additionally, the C-type lectins family plays a crucial role in innate immunity for shrimp (Thiel et al., 2009). Zhao et al. combined the recombinant LVCTL1 of L. vannamei with several structural proteins of WSSV and found that C-type lectins can inhibit the pathological effects of WSSV in blood cells from reducing the lethality of WSSV (Zhao et al., 2009). These findings assume that Hsp90 and C-type lectin 2 play a crucial immune role in persistent WSSV infection.

The pentose phosphate pathway, biosynthesis of amino acids, glycerophospholipid metabolism, and fatty acid biosynthesis were all enriched in terms of viral infections. These pathways may play a role in the replication of WSSV. Similar to the Warburg effect of mammalian viruses, WSSV-infected cells in shrimp seem to require more metabolic alternations than normal cells to meet the metabolic requirements of viral replication for energy and biogenic raw materials (I-Tung et al., 2011). The results showed that the pentose phosphate pathway (PPP) and amino acid biosynthesis were activated, and both played an essential role in the Warburg effect (Mei-An et al., 2014). As a marker of the Warburg effect, PPP provides nucleotides and nucleic acids for virion replication (Li et al., 2019). Amino acid biosynthesis directly provides raw materials for virus replication. In this study, WSSV may actively regulate the PPP and amino acid biosynthesis through the Warburg effect to provide raw materials and energy for its replication, which may be a critical factor in the long-term survival of WSSV in shrimp. Furthermore, fatty acid biosynthesis was activated in this study. Fatty Acid is an essential component of cells and participates in regulating a variety of biological processes. Hsieh et al. showed that WSSV 
induced the host to synthesize fatty acid through the PI3K-Akt-mTOR-HIF1alpha pathway, which provided bioenergetic for viral replication and promoted virions biosynthesis (Hsieh et al., 2015). Thus, fatty acid biosynthesis may also play a similar role in this study.

Among recent reports on human-pathogenic coronavirus, lipid metabolism remodeling was considered a critical link in replicating coronavirus in the host (Yan et al., 2019). Researchers detected that viral infection, such as the coronavirus, usually causes a rearrangement of lipid components in host cells (Hsu et al., 2010). The virus induces the host to make lipids for its envelope (Murillo et al., 2015), and the formation of the envelope creates a favorable condition for the invasion and proliferation of the virus (Lorizate et al., 2011). Therefore, glycerophospholipid metabolism was activated in this study. It is well known that the cell membrane components are mainly composed of glycerophospholipid components. Glycerophospholipid metabolism participates in lipid metabolism, which is a critical pathway of lipid metabolism. Therefore, we speculated that the glycerophospholipid metabolism in WSSV infected shrimp is probably like the lipid metabolism in coronavirus infected human cells: WSSV alters host lipid metabolism, rearranging lipid distribution in the host and promoting viral assembly. It is noteworthy that in the current coronavirus research, Yan et al. confirmed that exogenous supplementation of arachidonic acid (AA) and linoleic acid (LA) could destroy the lipid metabolism balance established by the virus and achieve the purpose of restricting the replication of coronavirus (Yan et al., 2019). At this point, it is worth further study whether AA and LA can be added in the study of WSSVinfected shrimp to achieve the purpose of treatment.

In this study, we found that following the spontaneous outbreak of WSSV, the surviving M. japonicus was under persistent WSSV infection. Our data shows that WSSV promotes replication and transcription using the material and energy required for average shrimp growth. WSSV changes the host biosynthesis and metabolic growth pathway and produces virions. In turn, M. japonicus regulated the Wnt signaling pathway and Ascorbate metabolism and synthesized $\mathrm{Hsp} 90$ and C-type lectin 2 to strengthen its immune responses against WSSV to resist further viral invasion. Maintaining a delicate balance between WSSV replication and host immunity enables the M. japonicus to carry low viral loads for longterm survival.

\section{Acknowledgements}

This research was funded by the key research and development projects in Guangdong Province (Grant No. 2020B0202010009), the project of 2019 Annual Guangdong Provincial Special Financial Fund (Grant No. 231419025) and the Fangchenggang Science and Technology Plan Project (Grant No. AD19008017).

\section{References}

Altschul, S.F., Madden, T.L., Schäffer, A.A., Zhang, J., Zhang, Z., Miller, W., Lipman, D.J., 1997. Gapped BLAST and PSI-BLAST: a new generation of protein database search programs. Nucleic Acids Research 25(17): 3389-3402. https://doi.org/10.1093/nar/25.17.3389

Clevers, H., Nusse, R., 2012. Wnt/ $\beta-C a t e n i n$ Signaling and Disease. Cell 149(6): 11921205. https://doi.org/10.1016/j.cell.2012.05.012

Escobedo-Bonilla, C.M., Alday-Sanz, V., Wille, M., Sorgeloos, P., Nauwynck, H.J., 2010. A review on the morphology, molecular characterization, morphogenesis and pathogenesis of white spot syndrome virus. Journal of Fish Diseases 31(1): 1-18. https://doi.org/10.1111/j.1365-2761.2007.00877.x

Flegel, T.W., 2007. Update on viral accommodation, a model for host-viral interaction in shrimp and other arthropods. Developmental \& Comparative Immunology 31(3): 217-231. https://doi.org/10.1016/j.dci.2006.06.009

Flegel, T.W., 2009. Hypothesis for heritable, anti-viral immunity in crustaceans and insects. Biology Direct 4(1): 1-8. https://doi.org/10.1186/1745-6150-4-36

George, S.J., 2008. Wht Pathway A New Role in Regulation of Inflammation. Arteriosclerosis Thrombosis \& Vascular Biology 28(3): 400. https://doi.org/10.1161/ATVBAHA.107.160952 
He, Z., Zhao, J., Liao, X., Chen, X., Ni, Z., 2020. The secondary bacterial infection caused by WSSV outbreaks impacts shrimp Marsupenaeus japonicus' growth as well as its intestinal microbiota's composition and function. The Israeli journal of aquaculture Bamidgeh 72. https://doi.org/10.46989/001c.21687

Hsieh, Y.C., Chen, Y.M., Li, C.Y., Chang, Y.H., Liang, S.Y., Lin, S.Y., Lin, C.Y., Chang, S.H., Wang, Y.J., Khoo, K.H., Aoki, T., 2015. To complete its replication cycle, a shrimp virus changes the population of long chain fatty acids during infection via the PI3K-AktmTOR-HIF1a pathway. Developmental and Comparative Immunology 53(1): 85-95. https://doi.org/10.1016/j.dci.2015.06.001

Hsu, N.Y., Ilnytska, O., Belov, G., Santiana, M., Chen, Y.H., Takvorian, P.M., Pau, C., Schaar, H., Kaushik-Basu, N., Balla, T., 2010. Viral Reorganization of the Secretory Pathway Generates Distinct Organelles for RNA Replication. Cell 141(5): 799-811. https://doi.org/10.1016/j.cell.2010.03.050

Chen, I.T., Aoki, T., Huang, Y.T., Hirono, I., Chen, T.C., Huang, J.Y., Chang, G.D., Lo, C.F., Wang, H.C., 2011. White Spot Syndrome Virus Induces Metabolic Changes Resembling the Warburg Effect in Shrimp Hemocytes in the Early Stage of Infection. Journal of Virology 85(24): 12919-12928. https://doi.org/10.1128/JVI.05385-11

Leu, J.H., Lin, S.J., Huang, J.Y., Chen, T.C., Lo, C.F., 2013. A model for apoptotic interaction between white spot syndrome virus and shrimp. Fish \& Shellfish Immunology 34(4): 1011-1017. https://doi.org/10.1016/j.fsi.2012.05.030

Li, C., Weng, S., He, J., 2019. WSSV-host interaction: Host response and immune evasion. Fish \& Shellfish Immunology 84: 558-571. https://doi.org/10.1016/j.fsi.2018.10.043

Li, F., Xiang, J., 2013. Recent advances in researches on the innate immunity of shrimp in China. Developmental \& Comparative Immunology 39(1-2): 11-26. https://doi.org/10.1016/j.dci.2012.03.016

Lightner, D.V., 2011. Virus diseases of farmed shrimp in the Western Hemisphere (the Americas): A review. Journal of Invertebrate Pathology 106(1): 110-130. https://doi.org/10.1016/j.jip.2010.09.012

Lorizate, M., Krusslich, H.G., 2011. Role of Lipids in Virus Replication. Cold Spring Harbor Perspectives in Biology 3(10): a004820. https://doi.org/10.1101/cshperspect.a004820

Love, M.I., Huber, W., Anders, S., 2014. Moderated estimation of fold change and dispersion for RNA-seq data with DESeq2. Genome biology 15(12): 550. https://doi.org/10.1186/s13059-014-0550-8

Mei-An, Su, Yun-Tzu, Huang, I-Tung, Chen, Der-Yen, Lee, Yun-Chieh, Hsieh. 2014. An Invertebrate Warburg Effect: A Shrimp Virus Achieves Successful Replication by Altering the Host Metabolome via the PI3K-Akt-mTOR Pathway. PLoS Pathogens 10(6): e1004196. https://doi.org/10.1371/journal.ppat.1004196

Murillo, A., Vera-Estrella, R., Barkla, B.J., Méndez, E., Arias, C.F., 2015. Identification of Host Cell Factors Associated with Astrovirus Replication in Caco-2 Cells. Journal of Virology 89(20). https://doi.org/10.1128/JVI.01225-15

Rao, R., Bing, R., Bhassu, S., Alinejad, T., Wang, J., 2015. A transcriptome study on Macrobrachium rosenbergii hepatopancreas experimentally challenged with white spot syndrome virus (WSSV). Journal of Invertebrate Pathology 136: 10-22. https://doi.org/10.1016/j.jip.2016.01.002

Santos, C.A., Andrade, S., Fernandes, J., Freitas, P.D., 2020. Shedding the Light on Litopenaeus vannamei Differential Muscle and Hepatopancreas Immune Responses in White Spot Syndrome Virus (WSSV) Exposure. Genes 11(7): 805-. https://doi.org/10.3390/genes11070805

Sato, S., Fujita, N., Tsuruo, T., 2000. Modulation of Akt kinase activity by binding to Hsp90. Proceedings of the National Academy of Sciences of the United States of America 97(20): 10832-10837. https://doi.org/10.1073/pnas.170276797 
Song, K.K., Li, D.F., Zhang, M.C., Yang, H.H., Ruan, L.W., Xu, X., 2010. Cloning and characterization of three novel WSSV recognizing lectins from shrimp Marsupenaeus japonicus. Fish \& Shellfish Immunology 28(4): 596-603. https://doi.org/10.1016/j.fsi.2009.12.015

Tassanakajon, A., Rimphanitchayakit, V., Visetnan, S., Amparyup, P., Somboonwiwat, K., Charoensapsri, W. Tang, S., 2018. Shrimp humoral responses against pathogens: antimicrobial peptides and melanization. Developmental and Comparative Immunology 80: 81-93. https://doi.org/10.1016/j.dci.2017.05.009

Tewary, A., Patra, B.C., 2008. Use of vitamin C as an immunostimulant. Effect on growth, nutritional quality, and immune response of Labeo rohita (Ham.). Fish Physiology \& Biochemistry 34(3): 251-259. https://doi.org/10.1007/s10695-007-9184-z

Thiel, S., Gadjeva, M., 2009. Humoral pattern recognition molecules: mannan-binding lectin and ficolins. Target Pattern Recognition in Innate Immunity 653(653): 58-73. https://doi.org/10.1007/978-1-4419-0901-5_5

Weiqing, W., Aijie, L., Siugin, C., 2002. Effects of dietary vitamin C on the immune function of shrimps, Penaeus chinensis. Journal of Ocean University of Qingdao 1(1). https://doi.org/10.1007/s11802-002-0030-8

Yan, B., Chu, H., Yang, D., Sze, K.H., Lai, P.M., Yuan, S., Shuai, H., Wang, Y., Kao, R.Y.T., Chan, J.F.W., Yuen, K.Y., 2019. Characterization of the Lipidomic Profile of Human Coronavirus-Infected Cells: Implications for Lipid Metabolism Remodeling upon Coronavirus Replication. Viruses 11(1). https://doi.org/10.3390/v11010073

Yuquan, L., Jiang, L., Wang, R., 2014. Layered farming for Marsupenaeus japonicus Bate. Chinese Journal of Oceanology \& Limnology 32(003): 549-553. https://doi.org/10.1007/s00343-014-3112-8

Zhao, Z.Y., Yin, Z.X., Xu, X.P., Weng, S.P., Rao, X.Y., Dai, Z.X., Luo, Y.W., Yang, G., Li, Z.S., Guan, H.J., 2009. A novel C-type lectin from the shrimp Litopenaeus vannamei possesses anti-white spot syndrome virus activity. Journal of Virology 83(1): 347-356. https://doi.org/10.1128/JVI.00707-08

Zhu, F., Zhang, X., 2013. The Wnt signaling pathway is involved in the regulation of phagocytosis of virus in Drosophila. Scientific Reports 3(1): 1-9. https://doi.org/10.1038/srep02069 